Théologiques

Théologiques

\title{
Le sanctuaire de l'Amour au coeur du territoire. Le territoire et le sacré dans la tradition judéo-chrétienne
}

\section{Marcelo Barros}

Volume 16, numéro 1, 2008

Le territoire et le sacré

URI : https://id.erudit.org/iderudit/019183ar

DOI : https://doi.org/10.7202/019183ar

Aller au sommaire du numéro

\section{Éditeur(s)}

Faculté de théologie et de sciences des religions, Université de Montréal

ISSN

1188-7109 (imprimé)

1492-1413 (numérique)

Découvrir la revue

Citer cet article

Barros, M. (2008). Le sanctuaire de l'Amour au coeur du territoire. Le territoire et le sacré dans la tradition judéo-chrétienne. Théologiques, 16(1), 25-38.

https://doi.org/10.7202/019183ar
Résumé de l'article

La tradition biblique à propos de la terre est ici relue à partir des enjeux environnementaux et des luttes des paysans et des peuples autochtones pour l'accès à celle-ci vue, non plus comme simple propriété et objet de dispute socio-économique, mais d'abord comme don et "sacrement » de la maternité divine. Les cultures du Proche-Orient ancien liaient le rapport au territoire au culte du dieu séjournant dans le sanctuaire du lieu. L'auteur retrace alors l'itinéraire singulier du peuple hébreu : son habitation d'une terre qui reste toujours promise, et son rapport à Dieu impliquant l'instauration de la justice. Dans le Deuxième Testament, le Christ constitue l'héritage promis, non comme substitut de la terre, mais comme " porte " et " pasteur " vers le Royaume, vers " un ciel nouveau et une terre nouvelle où habitera la justice » $(2 \mathrm{P} 3,13)$. La conclusion dégage, pour le contexte actuel, quelques implications libératrices d'un lien resserré autrement entre le territoire et le sacré. 


\title{
Le sanctuaire de l'Amour au cœur du territoire
}

\author{
Le territoire et le sacré dans la tradition \\ judéo-chrétienne ${ }^{1}$
}

Marcelo BARROS*

En Amérique latine, toutes les fois que les agriculteurs et les indigènes s'organisent pour conquérir leur indépendance ou pour s'assurer la propriété de leurs territoires ancestraux, les Églises chrétiennes et les religions auxquelles ils appartiennent sont appelées à collaborer à ces mouvements de libération et de revalorisation des cultures autochtones, grâce à une "théologie de la terre et de l'eau ${ }^{2}$ » qui rend possible une transformation du rapport entre l'être humain et la Terre. Il s'agit d'une vision spirituelle où on ne voit plus simplement la Terre comme une propriété et un objet de dispute socio-économique, mais principalement comme symbole et sacrement de la maternité divine. Cela se produit actuellement aussi bien chez les populations qui habitent la forêt que chez celles qui vivent dans les régions semiarides, les montagnes et les plaines côtières. Partout sur le continent se développe la dévotion à Pacha-mama, un nom originaire des Andes, connu et vénéré également dans d'autres cultures et en des termes semblables pour désigner la Terre Mère en tant qu'expression affectueuse de la présence divine. Les religions indigènes et les cultes issus de l'Afrique développent

* Marcelo Barros, moine bénédictin et bibliste, est membre de la Commission théologique latino-américaine de l'ASETT (Association œcuménique des théologiens du Tiers-Monde) et secrétaire de la Pastorale de la Terre et des mouvements populaires du Brésil. Il est l'auteur de 32 livres et collabore à diverses revues latino-américaines et européennes.

1. Article traduit du portugais par Marc Girard.

2. On notera qu'il s'agit du thème du Troisième Forum mondial Théologie et Libération qui se tiendra à Belem, Brésil, en janvier 2009, immédiatement avant le Forum social mondial. 
d'une manière naturelle et profonde cette spiritualité d'amour envers la Terre et envers tous les êtres vivants.

Pour des motifs historiques, la tradition judéo-chrétienne n'a pas la même facilité pour contempler Dieu dans les forces de la nature et dans la terre. En fin de compte, dans les sociétés du Proche-Orient ancien, les religions de la nature légitimaient le système social injuste qui faisait souffrir les Hébreux. Il importe de ne pas oublier que certains pharaons d'Égypte attribuaient leur richesse et leur pouvoir de conquête à Aton-Rê, le soleil, et qu'on les considérait comme fils du soleil. Le roi de Babylone, lui aussi, recevait son pouvoir des forces de la nature. Et les rois cananéens eux-mêmes se considéraient comme les privilégiés de leurs dieux. Pour que les Hébreux puissent se libérer de l'esclavage imposé par les rois de Canaan, d'Égypte et de Mésopotamie, ils ont été obligés de rompre avec les cultes de la nature et de la terre symbolisées, dans leur contexte, par les divinités cananéennes et phéniciennes. Ils ont insisté sur le fait que le Dieu de la Bible se manifeste avec plus de force et de visibilité dans l'histoire et les événements, bien qu'ils n'aient jamais nié que la terre constitue le premier sacrement et sanctuaire de Dieu. Nous allons approfondir ce thème et en tirer certaines conséquences pour notre temps.

\section{Terre et territoire dans la langue du Premier Testament}

Dans la Bible hébraïque, il n'existe pas de nom commun et univoque pour désigner la terre et le territoire d'Israël. Généralement, on dit que le terme le plus commun est ha'eres qui veut dire «terre " au sens de territoire géographique et même de pays: ha'eres Israel. De fait, c'est le terme qui apparaît le plus dans la littérature rabbinique et qui finit par être consacré par l'actuel État d'Israël pour légitimer un prétendu droit au territoire « de Dan jusqu'à Bersabée $^{3}$ » et, aujourd'hui, pour réprimer le peuple palestinien et nier son droit à la terre. Dans la Bible, en fait, cette expression se trouve seulement onze fois et jamais chez les Prophètes, sauf chez Ézéchiel qui l'utilise pour désigner la situation future idéale après l'Exil (par exemple, Ez 27,12; $42,2 ; 47,18)$. Là, 'eres Israel revêt une dimension de «terre renouvelée et

3. Dan et Bersabée étaient les sanctuaires situés à l'extrême nord (Dan) et à l'extrême sud (Bersabée), qui marquaient les frontières du territoire, lequel était signalisé par le sacré,

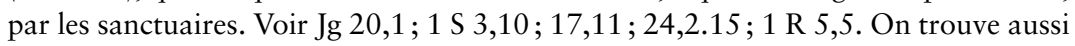
dans quelques textes bibliques l'expression «de la mer à la mer " pour délimiter les frontières de l'est à l'ouest (Ps 72). 
parvenue à sa plénitude ». Cela revient à parler de la terre comme symbole de la réalité future que le Nouveau Testament, certes, mais déjà quelques psaumes et certains prophètes, comme le Second Isaïe et les écrits apocalyptiques, appellent «Règne de Dieu ».

D'un autre côté, dans l'ensemble de la Bible, l'expression hébraïque la plus commune pour désigner la terre est 'ădāmâ qui a le sens de «sol fertile». Parfois, comme c'est le cas 19 fois, Ézéchiel utilise le terme, non pas en tant qu'entité politique ou patrie, mais plutôt comme milieu de vie, sol de la terre sainte et, dans certaines citations en contexte d'exil à Babylone, terre pauvre et abandonnée par Dieu ${ }^{4}$. De cette ‘ădāmâa, selon la Genèse, sera formé le 'àdām, l'être de terre qui a donné origine à l'humanité (Gn 2,7).

C'est cette terre qui constituera le premier sacrement de la promesse divine faite à Abraham et à ses descendants. Ainsi, dès les débuts des études historico-critiques, divers auteurs ont confirmé que la question de la terre était centrale dans tout le Premier Testament. Déjà à son époque, von Rad (1976, 81ss) constatait: «Dans tout l'Hexateuque, il n'existe aucun thème aussi constant et aucun motif théologique aussi fréquent que celui de la terre. La terre promise au début et donnée ensuite. [...] Pourtant, si étrange que cela puisse paraître, il n'y a pas encore d'étude sérieuse sur ce thème si central dans l'ensemble formé par les premiers livres bibliques. " À l'heure actuelle, il existe quelques études historiques et sociologiques sur la terre dans la culture biblique, mais il manque peut-être un pont commun ou mieux connu entre ces recherches et les théologies bibliques juives et chrétiennes qui en découlent. Voilà ce que, de manière simple et sans prétention de grande érudition, en dépit des limites restreintes de cet article, nous vous invitons à approfondir.

\section{Les sanctuaires qui délimitent la terre}

Dans une société de caractère sacral, comme l'étaient toutes les sociétés du Proche-Orient ancien, le droit d'un clan ou d'une tribu d'habiter un territoire déterminé était lié à l'adoration d'une divinité dans le sanctuaire qui réunissait la communauté des adorateurs de cette divinité. Le sanctuaire signifiait plus ou moins ce que serait, dans nos sociétés à nous, l'autorisation qu'un propriétaire donne à des agriculteurs de demeurer sur ses terres et de les cultiver. Le peuple vivait sur le territoire comme usufruitier ou locataire

4. Voir à ce sujet Keller (1975), cité dans González Lamadrid (1981, 25). 
de la divinité adorée au sanctuaire et qui était le véritable propriétaire de la terre. Beaucoup des récits patriarcaux des premiers livres de la Bible sont des récits étiologiques visant à justifier l'adoration de Dieu dans des sanctuaires cananéens comme Béthel (Gn 28), Sichem (Gn 12), Silo et d'autres. Ainsi, dès le début de l'histoire biblique, il existe une relation très profonde entre la terre et le sanctuaire. La présence d'un sanctuaire témoigne du fait que la terre est sacrée et appartient à Dieu. Les agriculteurs sont des usufruitiers sur la terre de Dieu et ils vivent une relation sacrée qui s'exprime non seulement dans l'adoration de ce Dieu, mais dans le rapport à la terre et aux diverses formes d'agriculture. Tout est sacré.

À l'intérieur de cette culture religieuse commune au Proche-Orient, l'histoire des Hébreux révèle quelques données nouvelles. Même si on ne connaît pas exactement l'origine de l'adoration yahviste, il est probable que le SEIGNEUR (YHWH) a été primordialement une divinité madianite du désert, et donc sans terre ni sanctuaire. Dans la culture en vigueur à l'époque, un dieu sans terre ni sanctuaire demeure sur le volcan éteint du Sinaï (Ps 68,18: « le SeIGneur vient du Sinaï»). C'est son habitat qui lui sert de temple. C'est pourquoi il peut promettre à Abraham et à ses descendants un territoire, même si ce territoire abrite déjà un temple dédié à une divinité cananéenne et est occupé par un clan de rois cananéens.

Il semble que le processus de la conquête du pays biblique se soit déroulé à travers certains épisodes de révolution paysanne, où les Hébreux ont conquis la terre des rois cananéens ${ }^{5}$. Mais en général, il s'est agi bien davantage d'un lent processus d'assimilation sociale, politique et religieuse. Au plan sociopolitique, les clans considérés comme formant Israël se sont peu à peu unis grâce à une foi commune et peu à peu renforcés comme peuple. Au plan religieux, il y a eu un syncrétisme fort entre les divinités des sanctuaires locaux et la foi nouvelle dans le SEIGneur (YHWH). Ainsi, Béthel, Silo, Dan, Mambré, Sichem et Jérusalem sont devenus toute la terre d'Israël ('eres Israel), propriété et sanctuaire du Seigneur.

Il est clair que les récits bibliques sur l'époque primitive et la conquête ont été écrits longtemps après et, comme tout l'indique, pour légitimer religieusement la conquête déjà réalisée (Barros et Caravias 1988, 139ss). Ces textes, lus au pied de la lettre, d'une manière fondamentaliste, peuvent légitimer conquêtes et oppression. Les Palestiniens d'aujourd'hui sont des témoins souffrants d'une pareille situation. Au contraire, l'insistance sur

5. Voir Gottwald (1986), surtout la partie IX: «Une nouvelle société cananéenne égalitaire: Israël libéré face aux peuples indigènes », p. 495; aussi Gottwald (1988). 
le fait que la terre appartient à Dieu a constitué une bonne base pour une théologie de la terre centrée sur la spiritualité écologique, aussi bien en rapport avec l'écologie de l'environnement qu'avec l'écologie sociale et humaine qui travaille pour promouvoir le droit des pauvres sans terre.

\section{Terre toujours promise mais partiellement atteinte}

Selon le récit biblique composé après l'exil à Babylone, toute l'histoire du peuple d'Israël commence par la promesse d'une terre pour Abraham et ses descendants. Cela a pour effet que le peuple qui relit ainsi son histoire est convaincu que son alliance avec Dieu dépend de la possession de cette terre. Faute d'habiter sur la terre donnée par Dieu, on ne peut pas vivre l'alliance intime avec lui. Cela entraîne deux conséquences.

Premièrement, la terre devient non seulement une question de culture agraire et de survivance, mais aussi de fidélité à l'alliance divine. Recevoir la terre et la conserver est un premier acte d'obéissance et de fidélité envers Dieu. J'ai observé moi-même une pareille manière de concevoir les choses chez les Indiens Pareci dans l'État du Mato Grosso du Sud. À la fin de la décennie '70, à un certain moment, ils souffraient d'une épidémie de coqueluche et les agents de la santé m'ont demandé d'aller parler aux anciens de la tribu qui refusaient la vaccination pour les enfants. J'ai essayé de convaincre les chefs. Mais ils m'ont dit: "Ça ne sert à rien de vacciner nos enfants. Cette maladie reflète la colère de Dieu parce que nous ne sommes pas morts en luttant quand les Blancs se sont emparés de notre terre, la terre que lui, Dieu, nous a donnée.»

Deuxièmement, la terre est l'héritage (nahală) de Dieu laissé à son peuple: elle désigne la propriété agricole pour l'homme et sa famille. Dans la Bible, le fait que la terre soit l'objet premier et fondamental de la promesse divine a diverses conséquences qu'on peut résumer comme suit.

1. La terre est don de Dieu à son peuple. Pour montrer que la terre est effectivement don de Dieu, les récits bibliques s'évertuent à montrer que la conquête aurait été pratiquement impossible si elle s'était appuyée seulement sur la force et la capacité des Hébreux. Les obstacles étaient nombreux et incontournables (Nb 13,27-33; Dt 1,26-28; 7,17-19; 9,1-6). Pourtant, les textes montrent aussi comment cette conquête si ardue s'est réalisée facilement: qu'il suffise de penser à la traversée du Jourdain que le peuple effectue à pieds secs (Jos 3,14-17), à la conquête de Jéricho, dont les murs tombent au son des trompettes (Jos 6); Dieu lui-même 
prend part à la lutte (Jos 10,11); même le soleil et la lune coopèrent à la victoire, arrêtant leur course pour assurer la victoire au peuple de Dieu (Jos 10,12-15).

La terre en tant que don est liée à la Torah. Quand le peuple obéit à la Torah et vit l'alliance avec Dieu, il conserve la terre. Quand il devient infidèle à l'alliance et aux commandements de la Torah, il perd la possession de la terre (Dt 6-8).

2. Si Dieu est le propriétaire de la terre, elle ne peut pas être vendue ni aliénée (Dt 11,10-12; Lv 25,23). Elle est l'espace où Dieu se manifeste, le témoin de l'alliance avec lui. Fréquemment, dans la Bible, le ciel et la terre sont invoqués comme témoins (Is 1,$2 ; 35,1$ ). Nombre de fois, la terre est une espèce de partenaire ou de collaboratrice de Dieu dans son projet d'amour. La terre dénonce le crime de Caïn; après, sous l'ordre de Dieu, elle le protège pour que personne ne le tue (Gn 4). Dans l'Exode, la terre collabore avec le peuple en fuite puis en marche au désert. De même, dans l'Apocalypse, le texte dit: "La terre vint au secours de la femme. La terre a ouvert sa bouche et avalé le fleuve que le dragon avait vomi » (Ap 12,16).

3. La terre est sacrée. Toute la terre (non seulement le territoire d'Israël, mais aussi le désert) est sacrée (Ex 3,4; Jos 5,15). «Le ciel est mon trône, et la terre, l'escabeau de mes pieds » (Is 66,1). Les prophètes se servent de cette parole de Dieu pour critiquer le Temple. La terre est le véritable temple de Dieu. "L'univers entier ne peut me contenir. Comment pour-

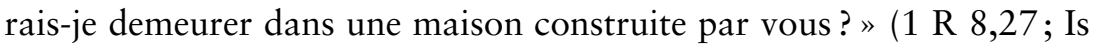
66) Pour les prophètes, le temple de Jérusalem est une sorte de résumé de l'univers et il est symbole de la sainteté de toute la terre. Il existe une relation intime entre le temple et la terre. Quand on conquiert la terre, on peut construire le temple - depuis l'époque des patriarches, un sanctuaire indique que le peuple a le droit de vivre sur cette terre et de la cultiver. Quand on perd la terre, on perd aussi le temple. Et toute la terre est profanée. C'est ce qui se produit au temps de l'Exil et de la captivité (Jr 7,26; Ez). Il faut que le peuple recouvre sa liberté pour que la gloire de Dieu revienne habiter dans le territoire et que ce territoire soit pleinement sanctuaire divin. Bien des fois au cours de l'histoire, dans une culture où la question de la propriété ne se posait pas de la même manière qu'aujourd'hui, le fait que le peuple se soit à ce point préoccupé de contrôler la possession de la terre signifiait que, pour le peuple d'Israël, s'assurer la possession de la terre était la manière d'éviter la profanation des lieux saints 
et, donc, des symboles les plus importants de l'alliance divine. Jérusalem est une cité-temple et, en ce sens, elle implique une certaine culture de ville, mais elle est en même temps symbole de toute la terre sainte.

La sacralité de la terre exige une attention spéciale empreinte de respect et de vénération. Comme en beaucoup de milieux indigènes d'Amérique latine, on considère que la terre est profanée si on y verse le sang. Déjà dans la Genèse, Dieu dit à Caïn : «De la terre, le sang de ton frère crie vers moi» $(\mathrm{Gn} 4,10)$. La terre profanée par le sang se venge, se ferme, ne donne plus ses produits et a besoin d'être à nouveau conquise (Ez 9,9). Les prophètes disent que la terre a plus besoin de justice que d'eau (Am 5,25).

4. La possession de la terre a des limites et exige le respect des autres. Non seulement la terre ne peut-elle être vendue, mais elle doit dans certains cas être partagée. "À chaque période de sept ans, tu feras remise [...] pour qu'il n'y ait pas de pauvres au milieu de toi » (Dt 15,1.4). Les prophètes condamnent l'accumulation des terres et ordonnent de retourner aux plus pauvres les champs et les propriétés (Mi 2,1-5; Ne 5,3-5.7-11; $\operatorname{Pr} 15,25)$. Même Israël doit respecter la terre des autres (Dt 2,5-10).

5. Il n'est pas permis de nuire à la terre, ni à sa propre terre ni à celle de l'ennemi. "Quand, pendant longtemps, tu assièges une ville pour t'en emparer, ne détruis pas ses arbres à coup de hache [c'était la coutume chez tous les peuples en guerre d'agir ainsi]. Tu pourras manger de leurs fruits. N'abats pas les arbres. Tu pourras seulement abattre les arbres qui ne portent pas de fruits, pour en faire des ouvrages de siège contre la ville ennemie » (Dt 20,19-20). D'après les récits bibliques de la conquête, le peuple d'Israël n'a pas toujours respecté cet ordre divin (2 R 3,24-25).

6. Dans cette promesse divine d'une terre, une promesse beaucoup plus large est contenue. La terre est plus qu'un symbole. Elle est comme le commencement de ce que, dans les évangiles synoptiques, Jésus appelle le "Royaume» ou Règne divin. En ce sens, la terre promise pointe vers une réalité jamais pleinement atteinte. Une fois conquise, la terre de Canaan est la terre que Dieu donne à son peuple, mais elle ne répond pas complètement à l'attente de la terre idéale où coulent le lait et le miel, où les animaux coexistent pacifiquement et où l'être humain se réconcilie avec tout l'univers (Is $11,6-11 ; 65,17 ; 66$ ).

Il y a un rapport essentiel entre la terre et la justice. Le prophète Michée, qui a vécu au VIII ${ }^{\mathrm{e}} \mathrm{s}$. avant notre ère, attribue la perte de la terre au fait que les latifundistes se sont emparés des terres des pauvres; puis tous, à leur tour, ils les perdent: Dieu intervient en leur enlevant la terre de son 
peuple (Mi 2,1-5). Isaïe concrétise encore davantage cette dénonciation de la concentration des terres entre les mains des latifundistes de son époque (Is 5,8-10). «Voici que ce crime, de prétendre accumuler pour soi plus de terres, est le premier péché, le péché fondamental, qui rompt l'alliance avec Dieu » (Sicre 1984, 262-270).

Déjà précédemment, j’ai fait allusion au prophète Ézéchiel. Il a lui-même vécu comme déporté (sans terre) pendant l'Exil à Babylone. C'est l'un des prophètes bibliques chez qui la question de la terre apparaît le plus centrale (Keller 1975). Il prêche que la terre est dévastée parce que le peuple a oublié la loi du Seigneur. Le territoire sacré d'Israël est devenu une "solitude désolée » $(\mathrm{Ez} 6,14 ; 33,28-29 ; 35,3.7)$. Mais, pendant l'Exil, le prophète promet une restauration du peuple et de la terre (Ez 38-39). Les violents, ceux qui s'appuient sur les armes, ne conquerront pas la terre (Ez 33,23-29). Le retour au pays sera une initiative de Dieu (Ez 34-37) et s'accompagnera d'une sorte de réforme nouvelle pour le partage du territoire (Ez 43 ; 48). En même temps que ces promesses concrètes, le style apocalyptique commence à se faire jour, et la terre nouvelle se confond avec le Temple nouveau et un temps d'alliance nouvelle...

\section{Le sens sacré de la Terre dans le Nouveau Testament}

À étudier la réalité sociale et politique des premières communautés chrétiennes - tant les communautés périphériques de l'Asie Mineure et de la Grèce, où Paul et son équipe de missionnaires itinérants ont œuvré, que les communautés qui ont donné naissance aux récits évangéliques -, on comprend que la question de la terre, si centrale dans le Premier Testament, n'a pas la même importance dans les premiers écrits chrétiens. Dans la réalité de la périphérie urbaine des cités grecques, y compris celles du premier siècle de notre ère (à ne pas confondre avec la culture urbaine actuelle), le problème de la terre ne se posait pas de la même manière qu'il s'était posé pour le peuple de Dieu dans le Premier Testament. Socialement et politiquement, la terre de la province de Palestine, comme les Romains l'appelaient, appartenait à l'Empire, et les agriculteurs qui y travaillaient payaient de lourds impôts à l'Empire. Les Juifs qui réussissaient à être propriétaires y parvenaient à coût de corruption et de collaboration avec le régime impérial, ce qui les isolait des autres Israélites. Au dire des Actes des Apôtres c'est symptomatique - , le jour de la Pentecôte, au moment où les membres de la communauté chrétienne de Jérusalem se sont convertis, ceux qui pos- 
sédaient une propriété la vendirent et déposèrent le montant aux pieds des apôtres pour l'usage commun (Ac 2,44-45; 4,36-37).

Avec les nombreuses diasporas subies par le peuple, le problème de l'identité culturelle et religieuse d'Israël, puis des disciples de Jésus, est lié au défi de la dispersion, de l'inculturation au monde grec: de la sorte, on tend à symboliser et même à spiritualiser les réalités auparavant très concrètes et matérielles. Cela devient si étonnant que Paul, dressant la liste des dons et bénédictions spéciales accordés par Dieu à Israël, ne mentionne pas la terre (Rm 9). L'héritage fondamental que Dieu donne à son peuple, c'est le Christ. Il est la réalisation de la promesse de Dieu. En ce sens, en tant que symbole ou sacrement, il occupe la place que prenait auparavant la terre. Non pas comme substitut, mais, comme le quatrième évangile le lui fait dire, "porte» et "pasteur» qui conduit les brebis vers des terres où elles trouvent abondance de nourriture et vie pour toutes et chacune (Jn 10). De la sorte, Jésus n'élimine pas la lutte pour la terre. Bien au contraire, il lui donne une signification nouvelle. On ne saurait oublier ni amoindrir la portée de ce que Jésus proclame dans cette béatitude: «Heureux les petits, car ils posséderont la terre» (Mt 5,4).

Selon Matthieu et Luc, Jésus serait né dans un village de paysans et aurait vécu à Nazareth, en pleine Galilée rurale. Son métier d'artisan n'excluait pas mais, au contraire, d'après les études sociologiques, impliquait une double tâche: être à la fois agriculteur en saison de plantation et de récolte et, de manière plus urbaine, être artisan entre les périodes de récolte.

Les évangiles montrent que Jésus envisage la terre, avec ses cycles et ses fruits, comme un instrument pédagogique, un sacrement du Royaume. Les paraboles de Jésus invitent à lire la Parole de Dieu tout autant dans la semence, le travail agricole, les arbres et toute la nature.

En Marc - il fait bon l'observer - , le terme préféré pour désigner le lac de Galilée est thalassa, "mer", et non limne, "lac». Il y a certainement, derrière cette désignation, une option théologique: pour le peuple de la Bible, la mer évoque les forces du mal à dominer (les armées qui envahissent viennent de la mer). Dans le fait d'appeler mer le lac de Galilée, on peut voir une manière de considérer la Galilée comme la région des autres, les extrémités de la terre.

En ce sens, la terre devient non plus un simple territoire, si importante qu'en soit la possession, mais bien une valeur culturelle: la terre de la justice. Dans l'un des derniers documents de ce qu'on appelle le Nouveau Testament, il est dit clairement: "Nous espérons, conformément à ce qui 
nous a été promis, un ciel nouveau et une terre nouvelle où habitera la justice» $(2 \mathrm{P} 3,13)$.

\section{Invitation à rétablir le mariage entre le ciel et la terre}

Parler de mariage entre le ciel et la terre relève du langage symbolique, mais il s'agit d'un thème profondément biblique. Dans toute sa profondeur, l'alliance entre Dieu et son peuple inclut les animaux, les plantes, la terre, l'eau et toute la nature (Os 2,16-23).

Malheureusement, en tentant de se libérer de certains mythes asservissants et d'une sacralisation aliénante, la tradition chrétienne a été de connivence avec une sorte de banalisation de la vie qui a rendu plus difficile la relation de respect entre l'être humain et la terre. En général, on voit dans la nature seulement son aspect utilitaire. On se voit soi-même et on voit la terre uniquement en rapport avec le travail. Une relecture de la Bible, enracinée dans une sensibilité écologique renouvelée, peut nous aider à retrouver cette dimension de mariage entre le ciel et la terre.

Dans la Bible, depuis l'expérience de l'Exil, les communautés racontent l'acte de la création dans un récit qui souligne, au centre de tout, le fait que Dieu a établi le sabbat comme un temps de liberté et de repos sacré. Le sens de la célébration du sabbat implique qu'on se conçoive soi-même, ainsi que la création, comme partenaires de l'alliance avec Dieu. Le sabbat est l'achèvement de la création: le repos festif. C'est la fête de la création, le couronnement de la création. Le sabbat réalise le mariage entre Dieu et la création ( $\operatorname{Lv} 25)$.

Selon les prophètes exiliques, quand le peuple a perdu sa terre, les cieux se sont fermés et il n’y a plus eu de prophétie. Le psalmiste se plaint: «Nous ne voyons plus nos symboles; il n’y a plus de prophètes, et personne ne sait jusqu'à quand» (Ps 74,9). Selon le Nouveau Testament, quand Jésus s'est laissé plonger dans les eaux du Jourdain, les cieux se sont ouverts et la terre a pu de nouveau écouter la voix du Père (Mc 1,10; Jn 1,29-34). Un monde qui, d'une manière peccamineuse, sépare l'être humain et la nature et veut transformer sa propre vie en marchandise, divise indubitablement le ciel et la terre. Le mariage du ciel et de la terre ne dépend pas de l'être humain, dans la mesure où il implique une unité qui surpasse tout, mais de fait, l'être humain a pour mission de servir de lien dans cette union. Il est comme le célébrant de ce mariage. Bien des fois, cependant, il a été l'instigateur d'un divorce désastreux pour tous. C'est pourquoi il est urgent que 
les personnes converties à un cheminement d'écologie spirituelle assument la mission d'être les agents de cette réconciliation.

Toute personne de bon sens perçoit que, si importantes que soient les solutions techniques et scientifiques, si nous ne créons pas une culture d'amour dans la relation de l'être humain avec la terre, l'eau et tous les êtres vivants, nous ne réussirons pas à surmonter la crise écologique grave qui atteint de nos jours la planète Terre.

Voilà pourquoi il importe de nous ouvrir le cœur au pluralisme que Dieu nous fait voir et d'apprendre, avec les religions indigènes et noires, la manière de contempler la présence divine dans le cosmos et principalement dans la Terre Mère.

Il importe aussi que les chrétiens redécouvrent, au sein de leur propre foi judéo-chrétienne, la dimension écologique de la spiritualité biblique et liturgique. Cet effort va nous enseigner à unir de nouveau création et rédemption, de même que la théologie de la libération a toujours insisté sur le fait qu'il existe une seule histoire et que, en elle, nous rencontrons l'action de Dieu qui unit création et libération. Un bon moyen de vivre cela consiste à revaloriser la dimension symbolique de la création, en unissant la terre, l'eau et tout le cosmos aux sacrements de la foi qui sont les sacrements du Règne de Dieu.

Comme "le chemin se fait en marchant », il faut redécouvrir la création comme temple de Dieu. Au temps de la conquête, les Espagnols ont dû construire, à Cuernavaca (Mexique), à côté de la cathédrale classique, une Église sans toit, parce que les Indiens refusaient de louer Dieu enfermés dans une bâtisse. Il nous faut retrouver cette relation entre liturgie et nature et faire de l'espace libre et vert un temple de louange et d'adoration. Il nous faut apprendre à embrasser les arbres, à récupérer avec les chamans la capacité d'entendre la parole divine des animaux et des plantes.

Au Brésil, les communautés ecclésiales de base ont redécouvert et utilisé dans beaucoup de célébrations le sacrement de la Terre, tout comme autrefois dans la culture du judaïsme: les exilés d'Israël avaient coutume d'emporter avec eux un peu de terre de leur pays pour que, où qu'ils soient, ils déposent sur le sol ce peu de terre qui venait de la terre promise et puissent prier en marchant dessus ou en s'y agenouillant. En Amérique latine, bien des fois nous avons fait la même chose dans des célébrations avec un peu de terre provenant de zones de conflits où les agriculteurs avaient donné leur vie pour la cause de la justice, ou dans des régions significatives pour nos populations pauvres, comme le Chiapas ou d'autres lieux semblables. 
On porte la terre dans les processions d'entrée et aussi au moment de la profession de foi. Les participants touchent la terre ou l'embrassent et renouvellent leur engagement en faveur de la justice et de la foi. De la même manière, on valorise l'eau: on a célébré les baptêmes par immersion ou, du moins, avec suffisamment d'eau pour bien mouiller; et toute la communauté, par après, touche l'eau ou en reçoit une aspersion. Les autres sacrements retrouvent également leur qualité de signe tout à la fois symbolique et réel.

De ce point de vue, il reste beaucoup à faire, un long chemin à parcourir.

Puisque dans cet article nous traitons la question du sacré en tant que symbole, il est bon de nous rappeler que l'hypothèse Gaia a été lancée par le scientifique anglais James Lovelock (1969) et complétée par William Golding, ce poète qui a suggéré le nom du mythe grec Gaia. Ainsi est-on arrivé à l'hypothèse Gaia. Que signifie cette théorie? Les scientifiques montrent que le système Terre est l'unique système biocybernétique qui a une tendance à l'homeostasis (maintien de conditions relativement constantes à travers un contrôle actif).

Il est important de percevoir que la biosphère de la terre, avec l'atmosphère, les océans et la terre, sont constitués en un système complexe et unique qu'on peut considérer comme un "organisme » sui generis, capable de maintenir la planète dans des conditions favorables à une vie assurée par la captation de l'énergie solaire. Il ne s'agit pas de mystifier ou de diviniser la terre, mais de la comprendre comme un système intelligent qui tend à créer des conditions de vie environnementales optimisées.

En conclusion de tout cela, disons que la Terre n'est pas seulement une force primitive à vaincre, ni une grande barque naviguant dans l'espace sans signification ni finalité. La Terre travaille comme un "superorganisme». Avec sa propre subjectivité, elle transforme les macromolécules, les microorganismes et les cellules sous forme de vie qu'ensuite elle conserve. Le langage Gaia est le code génétique, une langue universelle dont se servent toutes les cellules. Le système Terre dispose d'un système de sécurité efficace qui protège celle-ci d'associations génétiques dommageables pour la vie. Et il dispose d'une intelligence supérieure et d'une mémoire accumulée dans le cours de millions d'années. De nos jours, on parle d'une «sagesse du corps» (bodywisdom). Les populations qui entretiennent un plus grand rapport avec la terre ont une «culture onirique ».

En parlant de "sacré », nous parlons d'un acte d'amour. Dieu crée en se retirant lui-même des choses qu'il laisse exister en dehors de lui et pour 
leur propre compte, en se séparant de l'être humain qu'il laisse à sa propre responsabilité. Nous croyons que Dieu aime sa création et, en elle, favorise tout ce qui vit. De l'alliance de Dieu avec l'humanité et avec la création dérivent les droits humains et les droits de la nature. La Terre et la nature ne sont pas subordonnées aux humains, mais partenaires d'alliance avec Dieu. Vivre une option de communion avec le peuple pauvre qui, sur terre, souffre des injustices du système social et économique actuel, et rattacher la lutte pour la justice agraire à la justice socio-environnementale, c'est vivre l'amour divin et le renouvellement de l'alliance biblique. Comme disait saint Augustin: "Qu'on me montre quelqu'un qui aime, il va sentir ce que je dis. Qu'on me donne quelqu'un qui désire, qui marche dans ce désert, quelqu'un qui a soif et soupire après la fontaine de la vie. Qu'on me montre cette personne-là, et elle saura ce que je veux dire» (Traité sur l'évangile de Jean, 26,4).

\section{Références}

Barros, M. et Caravias, J.L. (1988), Teologia da Terra, Petrópolis, Vozes.

Keller, B. (1975), «La Terre dans le livre d'Ézéchiel », Revue d'Histoire et de Philosophie Religieuse, 55, p. 481-490.

Gonzalez Lamadrid, A. (1981), La Fuerza de la Tierra, Salamanca, Sigueme.

GotTwald, N.K. (1986), As tribos de Iahweh. Uma sociologia da Religião de Israel liberto, 1250-1050 A.C., São Paulo, Paulinas. [= (1979), The Tribes of Yahweh: A Sociology of the Religion of Liberated Israel, Maryknoll, Orbis]

(1988), Introdução Socioliteraria à Biblia Hebraica, Paulinas. [= (1985), The Hebrew Bible: A Socio-Literary Introduction, Augsburg, Fortress Press].

Lovelock, J. (1969), Gaia. A New Look at Life on Earth, Oxford.

RAD, G. Von (1976), Estudios sobre el Antiguo Testamento, Salamanca, Sigueme.

SiCRE, J.L. (1984), "Con los pobres de la Tierra», La justicia social en los profetas de Israel, Madrid, Cristianidad. 


\section{Résumé}

La tradition biblique à propos de la terre est ici relue à partir des enjeux environnementaux et des luttes des paysans et des peuples autochtones pour l'accès à celle-ci vue, non plus comme simple propriété et objet de dispute socio-économique, mais d'abord comme don et «sacrement » de la maternité divine. Les cultures du Proche-Orient ancien liaient le rapport au territoire au culte du dieu séjournant dans le sanctuaire du lieu. L'auteur retrace alors l'itinéraire singulier du peuple hébreu: son habitation d'une terre qui reste toujours promise, et son rapport à Dieu impliquant l'instauration de la justice. Dans le Deuxième Testament, le Christ constitue l'héritage promis, non comme substitut de la terre, mais comme "porte» et «pasteur»vers le Royaume, vers "un ciel nouveau et une terre nouvelle où habitera la justice» $(2$ P 3, 13). La conclusion dégage, pour le contexte actuel, quelques implications libératrices d'un lien resserré autrement entre le territoire et le sacré.

\section{Abstract}

This paper proposes a new reading of the biblical tradition's understanding of the concept of "land" in light of environmental questions as well as peasant and native peoples' struggles for land ownership, understood not merely as property and a source of socioeconomic conflicts but foremost as gift and "sacrament" of divine motherhood. Ancient Near-Eastern cultures linked the land to the cult of a god whose sanctuary was located therein. The author follows the singular itinerary of the Hebrew people who inhabit a promised land and whose relationship to God implies that justice be done. In the Second Testament, Christ becomes the promised inheritance, not as a substitute to the land, but as a pathway, a shepherd to the Realm, a "new heaven and a new earth, the home of righteousness" (2 Peter 3:13). In conclusion, the author derives a number of "liberating" consequences from his analysis for a contemporary interpretation of the link between the land and the sacred. 